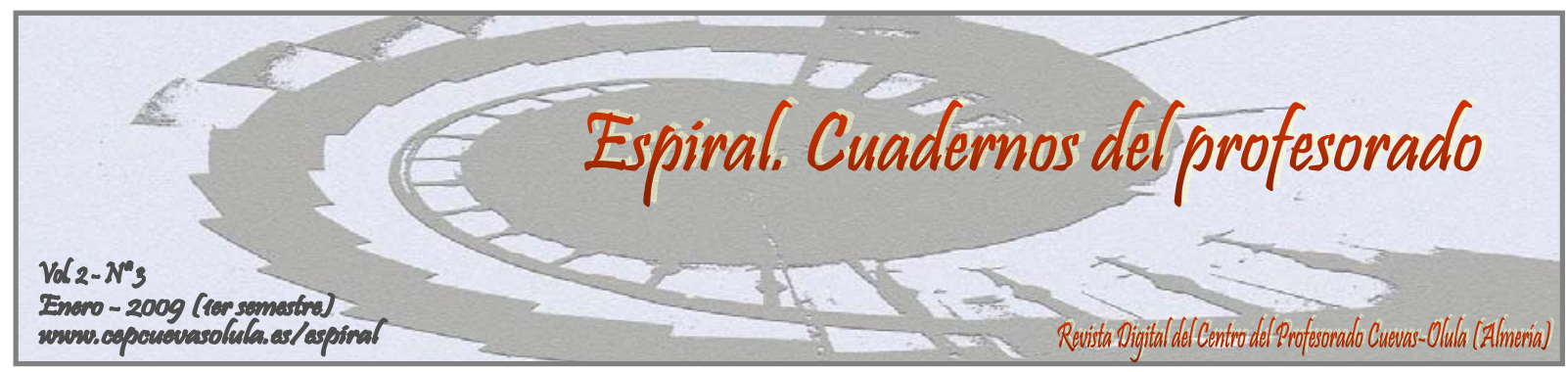

\title{
PROPUESTA DE INTERVENCIÓN PSICOLÓGICA EN LA MODALIDAD DEPORTIVA DE PIRAGÜISMO DE AGUAS TRANQUILAS
}

\section{PSICOLOGIST INTERVENTION IN FLATWATER CANOEING}

\author{
Fernando Alacid Cárceles ${ }^{(1)}$, Dr. Manuel Isorna Folgar ${ }^{(2)}$ \\ y Dr. Manuel Gómez López ${ }^{(1)}$ \\ (1) Facultad de Ciencias del Deporte. Universidad de Murcia (España) \\ ${ }^{(2)}$ Facultad de Educación y el Deporte. Universidad de Vigo (España)
}

Alacid Cárceles, F., Isorna Folgar, M. \& Gómez López, M. (2009). Propuesta de intervención psicológica en la modalidad deportiva de piragüismo de aguas tranquilas [en línea]. Espiral. Cuadernos del Profesorado, 2(3), 3-14. Disponible en: http://www.cepcuevasolula.es/espiral.

Enviar correspondencia a: mgomezlop@um.es

RESUMEN: Con este trabajo se pretende que a partir del análisis de una especialidad deportiva como en este caso ha sido el Piragüismo de aguas tranquilas y teniendo en consideración las diferentes situaciones que pueden llegar a darse a lo largo de toda una temporada o bien en la propia competición deportiva, ser capaces de elaborar un programa de intervención de entrenamiento psicológico para los deportistas de dicha modalidad, desarrollando las habilidades psicológicas que incidirán en el rendimiento deportivo.

Palabras clave: intervención, piragüismo, entrenamiento psicológico, motivación.

ABSTRACT: With this work it is intended that the analysis of a particular sport such as this case has been the calm water canoeing and taking into consideration the different situations that could occur throughout a season or competition sports, be able to develop an intervention program of psychological training for athletes of this modality, developing psychological skills that affect athletic performance.

Key words: intervention, canoeing, psychological training, motivation. 


\section{1.- INTRODUCCIÓN.}

Este trabajo se ha enfocado desde un análisis de la especialidad deportiva de piragüismo de aguas tranquilas y a partir de las diversas situaciones que pueden darse en la competición, elaborar un programa de intervención de entrenamiento psicológico para los palistas de esta modalidad deportiva. En dicho programa, se intenta desarrollar las habilidades psicológicas que podemos intuir como más útiles para el rendimiento deportivo. La principal dificultad del trabajo consiste en saber ajustar la planificación de una temporada a las distintas necesidades del entrenamiento psicológico.

Partiremos analizando la lógica interna del piragüismo de aguas tranquilas, para de esta forma diseñar un programa de intervención lo más ajustado posible a los requerimientos del deportista.

\section{2.- CARACTERÍSTICAS DEL PIRAGÜIS- MO DE AGUAS TRANQUILAS.}

Nos encontramos ante un deporte en el que existen dos modalidades principales: kayak (el palista se encuentra sentado y palea de forma alternativa por ambos lados) y canoa (el palista apoya una rodilla en la embarcación y palea por un solo lado). En kayak existe modalidad masculina y femenina, mientras que en canoa únicamente hay modalidad masculina.

Se compite sobre distancias de 200, 500 y 1000 metros, en una pista acotada por boyas que delimitan un total de nueve calles en línea recta. El reglamento prohíbe que las embarcaciones se acerquen mientras que se encuentran compitiendo, ya que podrían beneficiarse de la estela producida por el adversario, suponiendo un ahorro energético aproximado de un 11\% (PérezLandaluce, Rodríguez-Alonso, FernándezGarcía, Bustillo-Fernández, \& Terrados, 1998). Se puede competir en embarcaciones individuales, dobles y cuádruples. Un mismo palista puede participar en diferentes embarcaciones y distancias. Por todo ello podemos considerar al piragüismo de aguas tranquilas como un deporte cerrado y en algunos casos de colaboración.

Las competiciones se organizan a lo largo de varios días, habitualmente los deportistas deben de realizar varias regatas en una misma mañana o tarde, ya sea para una sola prueba, o porque esté inscrito en varias.
La duración de las pruebas va desde algo menos de cuarenta segundos para el 200 metros a los cuatro minutos que pueden realizar las embarcaciones más lentas sobre los 1000 metros.

Un aspecto importante es la posible influencia de las condiciones meteorológicas sobre la competición y especialmente del viento.

\section{1.-CARACTERÍSTICAS FISIOLÓGICAS DEL PIRAGÜISMO DE AGUAS TRAN- QUILAS.}

Pese a las diferencias en cuanto a las distancias de competición, podemos considerar al piragüismo como un deporte de fuerza resistencia en el que para conseguir un buen rendimiento es necesario una buena capacidad aeróbica, eficiencia aeróbica en el umbral anaeróbico, y capacidad anaeróbica láctica, especialmente la tolerancia al lactado. Existen estudios que indican que los mejores palistas suelen obtener buenos resultados en todas las distancias de competición (Fry \& Morton, 1991).

La valoración del gasto energético la valoraremos siguiendo en función de los indicadores externos e internos:

$\square$ Indicadores externos: en este caso la distancia recorrida siempre es fija y por otro lado el tiempo empleado en recorrerla como indicador del rendimiento. Además se podría valorar la distribución del esfuerzo en la competición y la frecuencia de ciclo.

$\square$ Indicadores internos: frecuencia cardiaca, concentración de lactato, consumo de oxígeno, etc.

Respecto a los indicadores externos, la distancia recorrida es un aspecto invariable en competición, pero los deportistas pueden tener preferencias o aptitudes por una u otra distancia de competición, aspecto importante a conocer por el entrenador para realizar la distribución de los palistas en las diferentes embarcaciones y distancias.

Los indicadores internos (frecuencia cardiaca, concentración de lactato, consumo de oxígeno, etc.) encuentran todos relacionados con la intensidad del esfuerzo y con la distribución del mismo a lo largo de la distancia. Existen varias investigaciones que estudian la distribución del esfuerzo a lo largo de la competición de aguas tranquilas (Alacid \& Carrasco, 2004; Bishop, Bonetti, \& Dawson, 2002; Issurin, 1998) y alguna otra que añade a 
este análisis la evolución de las diferentes variables cinemáticas: velocidad, frecuencia de ciclo, longitud de ciclo e índice de ciclo (Alacid, Ferrer, Martínez \& Carrasco, 2005; Alacid et al., 2008). Las aportaciones principales de las mismas es que se compite principalmente con un planteamiento regresivo en velocidad y frecuencia de ciclo, siendo éste generalizado para todas las distancias y embarcaciones. Este es un aspecto importante, ya que podemos planificar la mejor distribución del esfuerzo para que nuestro deportista consiga el mejor rendimiento posible independientemente de la posición que lleve a lo largo de la carrera.

\section{3.- PRINCIPALES PUNTOS DEL PROGRA- MA DE INTERVENCIÓN.}

Para el desarrollo del siguiente punto, estableceremos las pautas y la temporalización de un entrenamiento psicológico a lo largo de la temporada. Sería interesante que la periodización del entrenamiento psicológico fuera elaborada por el conjunto de especialistas que trabajan junto al deportista, a saber, el entrenador, el psicólogo, el médico y el fisioterapeuta, entre otros. Es fundamental que a la hora de planificar este entrenamiento se tenga un alto conocimiento de las características propias del deporte para analizar los requisitos psicológicos del deportista.

\subsection{FASES DEL ENTRENAMIENTO PSICOLÓGICO.}

Según Balaguer y Castillo (1994), las fases son las siguientes:

$\square$ Evaluación de las habilidades psicológicas y establecimiento de objetivos.

$\square$ Entrenamiento de habilidades
psicológicas.
$\square$ Pre-competición.
$\square$ Competición.
$\square$ Post-competición.

En cada una de estas fases, las habilidades que se incluyen y las técnicas que se utilizan para entrenarlas, dependen de variables tales como el deporte en cuestión y el periodo de tiempo durante el cual va a trabajar el psicólogo con el deportista o con el equipo y de las características y necesidades del deportista o del equipo.

El objetivo principal del programa de entrenamiento psicológico consiste en desarrollar planes de acción para que los deportistas consigan adquirir y mejorar una serie de habilidades psicológicas. Se desarrollarán desde una perspectiva educativa pretendiendo que los piragüistas adquieran las habilidades necesarias para poder afrontar con éxito las dificultades con las que se encuentren de forma que les permita rendir al máximo de sus posibilidades. También se pretende que la adquisición de habilidades psicológicas favorezca la salud y bienestar psicológico del deportista.

El entrenamiento psicológico se realizará en los períodos que los palistas descansan del entrenamiento físico, pero su aplicación tendrá lugar a lo largo de toda la semana.

A lo largo de las distintas fases del programa se analizarán el funcionamiento de los deportistas y/o del equipo en las diferentes situaciones importantes (entrenamiento, pre-competición, competición y post-competición); se realizará el establecimiento de objetivos para la temporada de trabajo y se diseñarán y entrenarán los planes de acción para conseguirlos.

Tabla 1.- objetivos generales de un plan de preparación en cada periodo de la temporada (Buceta, 1999)

\begin{tabular}{llll}
\hline \multicolumn{1}{c}{ Periodo preparatorio } & \multicolumn{1}{c}{$\begin{array}{c}\text { Periodo de } \\
\text { competiciones } \\
\text { secundarias }\end{array}$} & $\begin{array}{c}\text { Periodo de } \\
\text { competiciones } \\
\text { principales }\end{array}$ & Periodo de descanso \\
\hline - Desarrollar la motiva- & $\begin{array}{l}\text { - Máximo aprovecha- } \\
\text { ción básica. }\end{array}$ & $\begin{array}{l}\text { - Consecución y mante- } \\
\text { mimiento de la experiencia } \\
\text { competitiva para la pre- } \\
\text { "forma psicológica”. }\end{array}$ & $\begin{array}{l}\text { - Análisis del funciona- } \\
\text { miento psicológico du- } \\
\text { - Favorecer al máximo el la temporada. }\end{array}$ \\
$\begin{array}{l}\text { aprovechamiento del en- } \\
\text { trenamiento. }\end{array}$ & $\begin{array}{l}\text { paración psicológica de } \\
\text { los deportistas. }\end{array}$ & $\begin{array}{l}\text { - Prevención de dificul- } \\
\text { tades en la competición. }\end{array}$ & $\begin{array}{l}\text {-Planteamiento de nue- } \\
\text { vas necesidades. }\end{array}$ \\
- Sentar las bases para & - Prevención y control de & - Intervención en mo- & - Adquisición de nuevas \\
optimizar el funciona- & dificultades con vistas a & mentos de crisis. & habilidades. \\
miento mental más ade- & optimizar el rendimiento & & \\
lante. & en el siguiente periodo. & & \\
& - Desarrollo del autocon- & & \\
& trol.
\end{tabular}




\subsection{1.- EVALUACIÓN DE LAS HABILIDADES PSICOLÓGICAS Y ESTABLECIMIENTO DE OBJETIVOS.}

El establecimiento de metas a principio de temporada debe estar acorde con las posibilidades de nuestros deportistas. La fase más importante se enmarcaría en los meses de noviembre y diciembre ya que es el período preparatorio en el que se trabaja la preparación física general.

Posibles objetivos generales de un plan de preparación en cada periodo de la temporada (Buceta, 1999) (tabla 1).

La evaluación de las habilidades psicológicas se realizará de forma individualizada intentando conocer al deportista para elaborar un programa que se adapte a sus características. Es importante que el entrenador colabore en el proceso de evaluación puesto que él es quien mejor conoce los puntos fuertes y débiles de sus piragüistas. El psicólogo debe dar a conocer los beneficios del programa sin prometer que únicamente con el mismo se alcanzarán todos los objetivos incidiendo en que el rendimiento, podrá optimizarse dentro de sus posibilidades una vez dominado dicho programa.

En definitiva se pretende que el palista tome conciencia de la importancia del factor psicológico como vía para alcanzar el estado óptimo de rendimiento en competición, es decir, el deportista debe vivenciar las siguientes sensaciones:

- Relajación física.

- Ausencia de presión.

- Tranquilidad mental.

- Alta energía (determinación y positivismo)

- Diversión.

- Esfuerzo y lucha.

- Automatismo de la palada.

- Alerta.

- Focalización mental.

- Alta autoconfianza.

- Control de la situación.

En esta fase realizaremos dos tipos de evaluaciones, una del estado general de las habilidades psicológicas y otra de la evaluación de las mismas en diferentes situaciones que, al piragüista, se le puedan plantear en la competición.

\section{Evaluación general:}

1. Con el deportista: las habilidades que exploraremos en primer lugar son la relaja- ción, la concentración, la autoconfianza/autoeficacia, la activación y la visualización valiéndonos de los siguientes métodos: cuestionarios, entrevistas y observación directa.

2. Con el entrenador: el técnico aportará gran cantidad de información sobre la personalidad de los deportistas en múltiples situaciones así como los resultados obtenidos últimamente y su estado anímico actual.

3. Con el resto de los miembros del equipo de trabajo: son importantes las aportaciones que pueda realizar el médico, el masajista, su entorno familiar, los compañeros de equipo,...

\section{Evaluación en diferentes situaciones:}

Interesa conocer el comportamiento de los palistas durante los entrenamientos, en la convivencia diaria con sus compañeros de equipo, al igual que en la evaluación general los métodos empleados serán la observación directa principalmente, revisión de vídeos de competición, cuestionarios y entrevistas.

La información recogida nos permite finalizar esta fase confeccionando un perfil sobre el estado actual de las habilidades psicológicas del deportista en el periodo genérico (meses de noviembre y diciembre).

\section{Establecimiento de objetivos:}

La estructuración de la información se realizará comentando con el deportista y el entrenador cuales son las metas que tienen tanto a largo como a medio y corto plazo. Al realizar este análisis es esencial poner en común las expectativas del entrenador y del deportista, tanto en lo referente al entrenamiento, a las competiciones, como al desarrollo de habilidades psicológicas. Es conveniente programar de forma escalonada los objetivos a conseguir, así como las técnicas, ejercicios, etc. que se van a utilizar.

\subsection{2.- ENTRENAMIENTO DE LAS HABILIDADES PSICOLÓGICAS.}

Esta fase de programación psicológica la encuadraremos en los meses de enero y febrero en los que el entrenamiento empieza a ser más específico incluyendo perfeccionamiento técnico en el paleo y preparación física especial.

Hay una serie de variables a tener en cuenta por el psicólogo, como son el conocimiento de las habilidades que se van a entrenar, el tiempo para su consecución, número de sesiones semanales, la duración de las mismas y las 
técnicas y ejercicios que se van a utilizar para su consecución. Diferenciaremos entre una preparación psicológica general y otra específica.

\section{Preparación general:}

Desarrollada principalmente en el mes de enero, en el cual se pretende conseguir el dominio de habilidades psicológicas fundamentales: control emocional (relajación, activación), la concentración, la autoconfianza y la visualización. Las técnicas para la interiorización de estas habilidades deben practicarse de forma regular ya que sólo con el entrenamiento continuado se alcanzará el grado de ejecución adecuado y el nivel de eficacia deseado. Con respecto al orden de aprendizaje de las distintas habilidades, la primera de ellas debe ser la relajación puesto que esta es necesaria para en aprendizaje y práctica de la visualización; sin embargo la última habilidad que se domine será la concentración, ya que exige un control de otras habilidades (autoconfianza, autoactivación, etc,...). Concretamente (Bacon, 1989, citado por (Balaguer \& Castillo, 1994) propone el siguiente orden: relajación, monólogos positivos, autoactivación, visualización y concentración.

La autoconfianza se define como aquella fuerza que nos ayuda a realizar un movimiento, ejecutar un gesto o ganar. La autoconfianza siempre va unida a nuestra determinación, es decir, hasta dónde queramos llegar. Se trata de uno de los mejores pronósticos de éxito competitivo, ya que está íntimamente relacionada con la ejecución y con las estrategias que maneja el deportista para resolver las diferentes situaciones de su deporte. Esta variable afecta a los niveles motivacionales, al gasto energético, a las emociones y pensamientos positivos, a la capacidad atencional, a la habilidad para manejar la presión, a la resistencia física, y a la probabilidad de lesionarse físicamente.

La falta de autoconfianza en los propios recursos puede propiciar un déficit de motivación, entre otros. En estos casos, el deportista si tiene interés por la actividad, pero no confía en sus posibilidades para afrontar lo que se le exige con éxito, lo que repercute directamente en su motivación. La estrategia de intervención en estos casos no debe dirigirse a incrementar el interés del deportista por la actividad, sino en fortalecer su auto-confianza.

En el caso de palistas que tienen un déficit de interés, debemos utilizar el establecimiento de objetivos de resultado de cierta dificultad (por supuesto alcanzables), que despierten el interés de los deportistas.

Mientras que en los déficits por baja autoconfianza, utiliza objetivos de realización que el deportista perciba que puede alcanzar, siendo esta percepción el factor clave que debe perseguir esta estrategia, ejemplos:

$\square$ Establecer objetivos a corto plazo y alcanzables (está realizando 40 abdominales en 60", plantéale que dentro de 15 días hay que realizar 45 si entrenas según lo previsto).

$\square$ Antes de cada microciclo y sesión explicarles los objetivos a alcanzar y que se pretende con cada actividad.

$\square$ Debemos reforzar verbalmente (¡muy bien!, ¡estupendo!, ¡así me gusta!, etc.) los esfuerzos que realiza aunque no obtenga los resultados esperados.

$\square$ Es importante enseñarles a analizar el por qué de los errores para no volver a cometerlos, las consecuencias siempre son resultado de la suma de varias causas.

$\square$ El trabajo en conjunto con los entrenadores, padres e incluso profesores para generar entre todos esta auto-confianza suele dar buenos resultados.

Al entrenar por primera vez una técnica psicológica debe hacerse en situaciones de poca exigencia competitiva (estables), para ir en progresiva dificultad hasta llegar a la situación real.

\section{Preparación específica.}

Enmarcada en el mes de febrero, se pretende haga uso de las habilidades anteriores en pruebas o situaciones concretas, se trata de aplicar los conocimientos de la otra fase en la ejecución deportiva, la enmarcaremos en este mes para tener un periodo de tiempo suficiente de trabajo antes del Campeonato Nacional de Invierno en el que se puede empezar a practicar en una situación competitiva real. El palista deberá conocer su estado de activación, autoconfianza, etc. son los adecuados antes de efectuar la salida. Y tendrá automatizadas técnicas para adecuar su estado psicológico a la situación competitiva. Por ejemplo: visualización de la regata durante el calentamiento realizando series no demasiado largas a intensidades parecidas a la competición. 
El control de los pensamientos negativos (derrotistas, negativistas, etc.) conducen a emociones negativas y fomentan el aumento del nivel de activación del palista (manifestado a nivel motor y cognitivo), generando un circulo vicioso en el que la activación se propicia a sí misma. Todo este proceso conlleva un bajo rendimiento. Por su lado, los pensamientos positivos o apropiados conducen a emociones que capacitan al individuo para obtener un nivel de rendimiento óptimo. Así, encontramos que lo que la persona piensa llega a convertirse en una especie de "Profecía Autocumplida".

Bunker y Williams (1986) consideran que existe un modo de pensar distinto para los "vencedores". Los deportistas más destacados tienen confianza en sí mismos, lo cual es consecuencia de un pensamiento adecuado y positivo. Ante una competición o reto piensan que "pueden hacerlo bien ", no minimizan sus

habilidades, sino que consideran sus capacidades y también sus limitaciones de una manera realista, centran sus pensamientos en el modo en que han de enfrentarse a la tarea y no en preocupaciones acerca de su posible rendimiento.

Uno de los síntomas más eficaces para detectar su forma de pensar es a través de los comentarios que suelen realizar y su sustitución inmediata por otros positivos (tabla 2).

La influencia de la atención puede afectar significativamente al rendimiento de los deportistas en el entrenamiento y la competición, resultando decisiva en todo lo que conlleva: a) Estar alerta b) Recibir y asimilar información c) Analizar datos d) Tomar decisiones e) Actuar a tiempo f) Actuar con precisión.

Cualquiera que sea el objetivo de una sesión de entrenamiento deportivo, el papel de la atención tiene una gran trascendencia, pues es importante que los deportistas se centren tanto en el objetivo concreto de la sesión como en las demandas específicas de cada uno de los ejercicios o tareas que forman parte de la mima.

En ocasiones, los deportistas deben utilizar una atención más consciente hacia los estímulos externos o las respuestas propias que son más relevantes para rendir en la tarea; este tipo de atención, favorece que estén más alerta respecto a los aspectos que se consideren clave, en beneficio de la asimilación de información, la toma de decisiones y la ejecución. Resultará especialmente útil, cuando se trate de aprender o perfeccionar habilidades, pues sin atención consciente será imposible que los deportistas puedan mejorar.

En el anexo II aparecen una serie de técnicas o estrategias para mejorar los procesos atencionales y reducir aquellos que puedan impedir su consolidación.

\subsubsection{PRE-COMPETICIÓN.}

Podemos encuadrar esta fase de entrenamiento psicológico en los meses de marzo y abril, ya que en estos meses comienzan a realizarse controles de larga distancia, principalmente para preparar el Campeonato de Nacional de Invierno, las aspiraciones de este período consisten en la adquisición y perfeccionamiento de estrategias previas a la competición. Pretendemos que el palista sea capaz de afrontar con éxito situaciones precompetitivas.

\section{Ejemplo de un plan pre-competitivo en piragüismo:}

Un plan pre-competitivo consiste en una serie de actividades que contribuirán al rendimiento óptimo del piragüista para la competición,

Tabla 2.- Pensamiento positivo y pensamiento negativo.

PENSAMIENTO NEGATIVO

\begin{tabular}{|c|c|}
\hline $\begin{array}{l}\text { "No puedo creerlo ¡hace aire!, y esto a mi me } \\
\text { perjudica muchísimo. }\end{array}$ & $\begin{array}{l}\text { "A nadie le gusta el viento, pero yo puedo palear tan bien } \\
\text { como cualquier otro/a" }\end{array}$ \\
\hline $\begin{array}{l}\text { El de la calle } 8 \text { ha hecho una semifinal } \\
\text { impresionante, se le ve más tranquilo/a, no creo que } \\
\text { pueda estar a su altura. }\end{array}$ & $\begin{array}{l}\text { Tengo que centrarme en mí mismo/a, se que si realizo los } \\
\text { tiempos de los entrenamientos estoy en las primeras } \\
\text { posiciones. Además pensando en lo que hará el/a no me } \\
\text { ayudará, tengo que centrarme en lo que tengo que hacer } \\
\text { yo. }\end{array}$ \\
\hline $\begin{array}{l}\text { Todos/as están más fuertes que yo, como no llegue } \\
\text { al } 200 \text { m. con todos/as no tengo nada que hacer. }\end{array}$ & $\begin{array}{l}\text { Tengo que centrarme en mi mismo, en no perder tiempo } \\
\text { en la salida, así que voy a visualizar la parte más } \\
\text { importante de la competición "la salida”: posición del } \\
\text { tronco, brazo extendido, observo el árbitro... }\end{array}$ \\
\hline
\end{tabular}


ejemplo de estas actividades una correcta alimentación, trabajo de flexibilidad, monólogos positivos, diálogos con el entrenador, etc.

Lo ideal es combinar un calentamiento psicológico con el físico y encontrar un foco atencional adecuado, con el fin de conseguir:

1. Fortalecer los sentimientos y estar preparado para reforzar la confianza en uno mismo y en su preparación, es decir, tener confianza en el trabajo realizado durante la temporada.

2. Evitar la intrusión de pensamientos amenazantes, ya que podrían disminuir la confianza o impedir una buena ejecución. No dejándose influir por los comentarios que puedan hacer antes de la salida los demás palistas o entrenadores. Por ello es importante orientar la atención lejos de lo que nos preocupa.

3. Buscar un estado emocional positivo, un nivel de activación óptimo y un foco atencional orientado a conseguir el mejor resultado.

La habilidad mental es una capacidad (capacidad de imaginarse, de verse a si mismo/a realizando cualquier movimiento) que poseen muchos de los grandes deportistas. Esta habilidad les permite anticipar muchas situaciones, además de recibir un feed-back (retroalimentación) mayor que la de aquellos deportistas que no son capaces de verse a sí mismo/a mentalmente. Es una habilidad que se puede "aprender" y por lo tanto enseñar. La estimulación de esta habilidad en edades tempranas permite que en categorías superiores la tenga consolidada y se pueda beneficiar de la misma.

El entrenamiento en imaginación consiste en que se visualice a sí mismo en una situación concreta (ej. En la salida de una regata, realizando una mala salida, etc.), o bien realizando cualquier movimiento (ej. torsión del tronco, desplazamiento de la cadera, etc.).

La práctica en imaginación favorece que:

1. El deportista utilice su "atención interna" evitando así interferencias externas que pueden dificultar su concentración.

2. Facilita que se pueda realizar una observación microscópica de los detalles más relevantes (ej. Sonido de la megafonía en la salida, momentos de cansancio del más directo rival, o bien de la propia técnica, etc.) y que luego irán sobreaprendidos.
3. Posibilita que el deportista pueda detectar las posiciones y los movimientos habituales que deben ser modificados y sustituidos por unos nuevos.

4. La práctica en imaginación permite realizar más ensayos de las posiciones y movimientos nuevos que si sólo se utilizara la práctica real en las sesiones de entrenamiento o bien ahorrarnos muchas sesiones de entrenamiento real.

Es una técnica que también se puede utilizar en periodos de convalecencia por lesiones. El trabajo en imaginación se complementa muy bien con la grabación real (ej. "Trabajo técnica": a través de la pantalla de vídeo y según las correcciones que se le realizan sobre los fallos que comete debemos entrenarlo para que él mismo/a se visualice realizándolo correctamente).

En cuanto al mantenimiento de un nivel de activación óptimo y un foco atencional orientado a conseguir el mejor resultado la motivación es una variable que resulta crucial en este campo ya que muestra o indica el grado de interés del deportista por su actividad y/o las consecuencias que le genera ésta. Se trata de una variable decisiva por su incidencia en el compromiso de los deportistas por su actividad y con ello en sus conductas de adherencia, persistencia y tolerancia a la adversidad. También influye en el nivel de activación de los deportistas y en los procesos cognitivos que afectan a su estado emocional.

Algunos de los errores que con mayor frecuencia cometen los entrenadores con los más jóvenes son:

1. Pretender que el deportista se motive por objetivos inalcanzables o a muy largo plazo sin etapas intermedias. Debemos procurar establecer objetivos a corto plazo y alcanzable y llegando a un acuerdo con él de lo que debe realizar para alcanzarlo.

2. Al contrario que lo anterior, intentar motivar a los deportistas únicamente respecto a las tareas inmediatas, sin situar esta tareas en un contexto más amplio en el que se tenga en cuenta su repercusión a medio/largo plazo. Debemos procurar explicarle día a día que objetivo se persigue con la tarea que tiene que realizar.

3. No tener en cuenta otras variables psicológicas que pueden afectar a la motivación como el estrés o la autoconfianza. Debemos establecer con él los objetivos (no los 
debe establecer el entrenador solo) y los medios para alcanzarlos.

4. No considerar la situación global, sólo la deportiva. No debemos olvidar que el deportista tiene vida extradeportiva (estudios, amigos/as, ligues, etc.) que, por mucho que nos pese, les suelen dar tanta o más importancia que al piragüismo. Debemos hacerles ver que la práctica del piragüismo es compatible con todo lo demás.

5. Hablarles sólo de los beneficios y de los posibles éxitos. Debemos plantearles los costes que tiene la práctica del piragüismo, aunque los beneficios son muy superiores a los costes (sólo así entrenará).

Una nota interesante recogida en Cagigal (1981) es que la mayoría de los jóvenes participan en los deportes para divertirse, para aprender destrezas nuevas y para hacer nuevos amigos/as. La investigación ha demostrado que el ganar carece relativamente de importancia si se compara con éstas otras metas. Debes dejarles claro diariamente que no serán nunca perdedores si se emplean con el máximo esfuerzo.

\subsection{4.- COMPETICIÓN.}

Esta fase se desarrolla durante los meses de mayo a agosto, en los que están incluidos: Circuitos de Pista Nacionales, Competiciones Internacionales, Campeonato Nacional por Comunidades Autónomas, Juegos Olímpicos, Campeonato Nacional por Clubes...

El objetivo fundamental de esta fase consiste en la adquisición y perfeccionamiento de estrategias para la competición, lo que se pretende es que el deportista dé lo mejor de si mismo en estos eventos. Estas estrategias son, plan de focalización y refocalización.

El miedo al fracaso suele ser uno de los determinantes del abandono o bajo rendimiento de muchos deportistas (Buceta, 1998). Buena parte de culpa, en muchas ocasiones la tienen los propios entrenadores por que depositan expectativas muy altas en ellos/as y hacen que ellos/as mismos/as se planteen objetivos muchas veces inalcanzables. El planteamiento que sugerimos es que sea el propio palista el que con el tiempo establezca sus propios objetivos.

Para alcanzarlo sugerimos que se potencie en él/ella el razonamiento lógico y analítico, es decir, en el momento de haber finalizado el entrenamiento o regata debemos preguntarle
¿Qué has hecho bien? ¿Qué has hecho mal? ¿Qué has podido realizar mejor?, etc. y que él/ella responda. Con asesoramiento y comentarios posteriores de su entrenador es más probable que afiance y fije lo aprendido en ese día.

Suele suceder que se generen expectativas basándose en los resultados de la última regata 0 competición, incluso esto puede condicionar su motivación en los entrenamientos. En caso de que la última regata o control no haya salido como se esperaba, puede ser interesante que el propio palista busque las causas de esos malos resultados y a la vez las posibles soluciones para que no se vuelvan a repetir. Ante la aparición de comentarios negativos (del tipo: ¡... total... ya me gana...!, $; \ldots$ todos tienen mejores tiempos que yo..!, etc.) debemos corregir inmediatamente esa forma de pensar y que busque las causas y posibles soluciones del por qué de esa situación.

Como bien sabemos, el entrenador juega un papel muy importante en la vida del deportista (tanto en la deportiva como en la personal). La confianza en el entrenador se desarrolla por diversas vías, por ejemplo por los conocimientos que posee, por su experiencia, por su prestigio, por que sabe transmitir, etc. para aumentar esta confianza debemos procurar:

- Crear un clima afectivo positivo entre ambos (recuerda que no es sólo un deportista).

- Procura cumplir las promesas realizadas.

- La autoridad debe ser firme, pero a la vez respetuosa y aceptada. Ten paciencia y no te fijes sólo en lo que hace mal, debemos alentarlo a la mínima ocasión.

En los deportistas jóvenes es más fácil instaurar formas de pensamiento que cuando ya están formados. Las actitudes deben quedar bien arraigadas en estas edades.

La figura del entrenador-profesor es clave. La manera en que un entrenador organice las sesiones de entrenamiento, los criterios que utilice para agrupar a los deportistas, el estilo de liderazgo que emplee (autoritario-participativo), los parámetros que utilice para evaluar el éxito (resultado versus esfuerzo y mejora), el trato que dispense a los palistas, el empleo de recompensas, etc., influirá de forma decisiva en la motivación de los jóvenes deportistas.

Los jóvenes palistas necesitan ser evaluados por su mejora y esfuerzo y no sólo por su ejecución en comparación con los otros 
deportistas. Por ello algunas pautas que debe seguir el entrenador podrían ser las siguientes:

- Trabajar con él posibles comentarios de otros adversarios (o incluso compañeros/as) que lo menosprecien.

- Plantear la posibilidad de que los rivales durante el calentamiento parezca mucho "más fuertes” que él/ella.

- Conseguir que interiorice y exprese palabras de ánimo, pensamientos positivos, autoinstrucciones (ej. "estoy entrenando bien... voy a competir mejor", "sin medalla ya estoy... ahora a por ella", "soy... por lo menos... tan bueno como los demás", etc.).

- Durante los momentos previos a la salida que piense en las palabras de ánimo que los compañeros, entrenador, padres, "amigos/as" le van a dar si realiza bien la competición, lo bien que se a sentir si "se vacía a tope”., etc.

- Debe recordarle y debe trabajar con él la importancia de esforzarse al máximo: "Ganar no es lo único importante, pero esforzarse por lograrlo sí lo es. El éxito consiste en esforzarse al máximo para obtener la victoria”.

- Debe reforzar los esfuerzos y no exigir sólo resultados. Debe recompensar inmediatamente todas las situaciones donde se esfuerce "a tope".

\section{Plan de focalización.}

Consiste en desarrollar una serie de rutinas que rellenen los periodos de tiempo durante los cuales el palista es más propenso a perder la concentración. Para entrenar el foco atencional durante la competición debemos enseñar a nuestro palista a qué y en qué momentos debe prestar atención durante la situación deportiva. En el caso que nos ocupa una estrategia que podríamos utilizar sería: una hora antes de la competición palear durante diez minutos para relajarse, media hora de concentración en la regata a realizar, calentamiento en tierra y calentamiento en agua con visualización de la carrera y activación psicológica.

\section{Plan de refocalización.}

Consiste en elaborar un listado de factores que puedan distraer al piragüista e indicar el camino para encontrar de nuevo la concentración. A nivel práctico podría tratarse de: mirar a las calles anexas durante la competición, o perder el equilibrio sobre la embarcación por la acción de las olas en algún momento de la regata. Volveremos a encontrar la concentración buscando como objetivo el final de la calle.

\subsubsection{POST-COMPETICIÓN.}

Situaremos esta fase en los finales de agosto y principios de septiembre cuando el deportista tiene un alto nivel de desgaste físico y psíquico, dejando el resto de la temporada para "desconectar" de la actividad casi totalmente.

En esta fase se persigue un análisis de la interpretación del deportista de sus resultados en la competición, puesto que de esta opinión dependerá en gran medida su motivación futura, su autoeficacia en la tarea, etc.

\section{4.- REFERENCIAS BIBLIOGRÁFICAS.}

Alacid, F., \& Carrasco, L. (2004). Distribución del esfuerzo en piragüismo sobre 1000 metros. En Varios (Ed.), III Congreso de la Asociación Española de Ciencias del Deporte (CD). Valencia: Universitat de València.

Alacid, F., Ferrer, V., Martínez, E., \& Carrasco, L. (2005). Análisis cuantitativo de la técnica de paleo en kayakistas infantiles. Motricidad. European Journal of Human Movement, 13, 133-146.

Alacid, F., López-Miñarro, P. A., Ferragut, C., García, A., Ferrer, V., \& Martínez, I. (2008). Evolución y comparación de la velocidad, frecuencia, longitud e índice de ciclo sobre $200 \mathrm{~m}$, en palistas infantiles de diferentes modalidades. Motricidad. European Journal of Human Movement, 20, 15-27.

Bacon, T. (1989). La planification et l'intégration des programes de préparation mentale. Science du Sport, 10 (1).

Balaguer, I. \& Castillo, I. (1994). Entrenamiento psicológico en el deporte. En I. Balaguer (Ed.), Entrenamiento psicológico en el deporte. Valencia: Albatros Educación.

Bishop, D., Bonetti, D. \& Dawson, B. (2002). The influence of pacing strategy on VO2 and supramaximal kayak performance. Medicine \& Science in Sports \& Exercise, 34 (6), 1041-1047.

Buceta, J. M. (1998). Psicología del Entrenamiento Deportivo. Madrid: Dykinson.

Buceta, J. M. (1999). Intervención psicológica en el entrenamiento deportivo. Revista de psicología del deporte, 8 (1), 39-52. 
Bunker, L.K. \& Williams, J.M. (1986). Cognitive techniques for improving performance and building confidence. En Jean M. Williams (Ed.). Applied sport psychology. Personal growth to peak performance. Palo Alto, California: Manfield Publishing Company.

Cagigal, J. M. (1981). !Oh Deporte! Anatomía de un gigante. Valladolid: Miñón.

Fry, R.W. \& Morton, A.R. (1991). Physiological and kinanthropometric attributes of elite flatwater kayakists. Medicine \& Science in Sports \& Exercise, 23 (11), 1297-1301.
Issurin, V. (1998). Analysis of the race strategy of world-class kayakers. En V. Issurin (Ed.), Science \& practice of canoe/kayak highperformance training: selected articles in memory of junior world champion Nevo Eitan (pp. 27-39). Tel-Aviv: Elite Sport Department of Israel.

Perez-Landaluce, J., Rodriguez-Alonso, M., Fernandez-Garcia, B., Bustillo-Fernandez, E. \& Terrados, N. (1998). Importance of wash riding in kayaking training and competition. Medicine \& Science in Sports \& Exercise, 30 (12), 1721-1724.

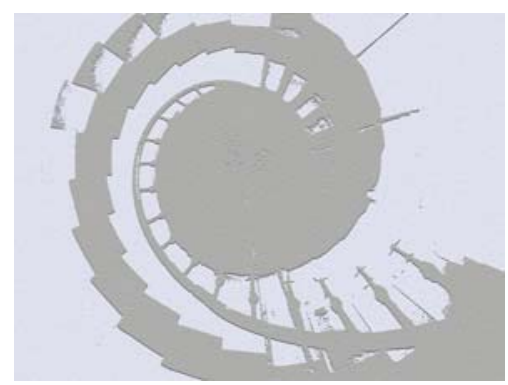


ANEXO I

\begin{tabular}{|c|c|c|c|}
\hline $\begin{array}{c}\text { OBJETIVO DEPORTIVO A } \\
\text { CONSEGUIR ( RESULTADO): }\end{array}$ & $\begin{array}{l}\text { PLAZO DE TIEMPO PARA } \\
\text { CONSEGUIRLO } \\
\text { - } \quad \text { Fecha actual: } \\
\text { - } \quad \text { Fecha límite: }\end{array}$ & 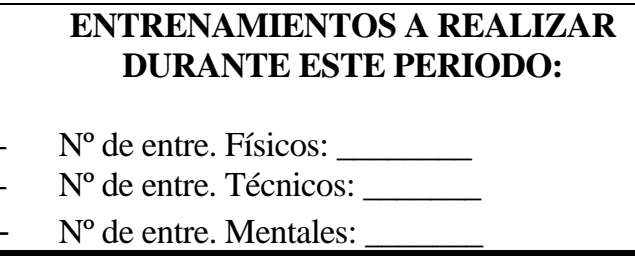 & $\begin{array}{l}\text { COMPETICIONES } \\
\text { CONTROLES) A REALIZAR EN } \\
\text { ESTE PERIODO: } \\
1 . \overline{ } \\
2 . \overline{ } \\
\text { 3. }\end{array}$ \\
\hline $\begin{array}{c}\text { ÁMBITOS A TRABAJAR PARA } \\
\text { CONSEGUIR EL OBJETIVO } \\
\text { DEPORTIVO } \\
\text { (OBJETIVOS DE REALIZACIÒN) } \\
\end{array}$ & $\begin{array}{l}\text { TRABAJO O TAREAS A REALIZAR } \\
\text { DENTRO DE CADA ÁMBITO }\end{array}$ & $\begin{array}{l}\text { POSIBLES DIFICULTDES QUE NOS } \\
\text { PODEMOS ENCONTRAR QUE } \\
\text { PUEDEN IMPEDIR EL ALCANZAR } \\
\text { LAS TAREAS PREVISTAS } \\
\end{array}$ & $\begin{array}{l}\text { SOLUCIONES A LAS POSIBLES } \\
\text { DIFICULTADES INTERFERENTES }\end{array}$ \\
\hline FISICOS: & & & \\
\hline TECNICOS: & & & \\
\hline TACTICOS/ESTRATEGI-COS: & & & \\
\hline PSICOLOGICOS: & & & \\
\hline OTROS: & & & \\
\hline
\end{tabular}

Con esta plantilla se pretende que juntos establezcáis un PLAN DE TRABAJO para conseguir un objetivo deportivo determinado. Decidido el objetivo de resultado a conseguir y conociendo el plazo de tiempo para lograrlo y los entrenamientos y competiciones de este periodo, se deben establecer las tareas a realizar (qué debe hacer) en los distintos ámbitos (físicos, tácticos/estratégicos, psicológicos y otros (que tú crees que son importantes para alcanzar el objetivo pactado), previendo las posibles dificultades que puedan surgir así como las posibles soluciones a las mismas 
ANEXO II

\begin{tabular}{|c|c|c|c|c|}
\hline & \multicolumn{2}{|c|}{ AUMENTAR ACTIVACION } & \multicolumn{2}{|c|}{ REDUCIR ACTIVACION } \\
\hline & ANTES DE LA COMPETICION & $\begin{array}{l}\text { DURANTE LA } \\
\text { COMPETICION }\end{array}$ & ANTES DE LA COMPETICION & DURANTE LA COMPETICION \\
\hline $\begin{array}{l}\text { ACTIVACION } \\
\text { FISIOLÒGICA }\end{array}$ & $\begin{array}{l}\text { - Ejercicio físico activador } \\
\text { - Autoinstrucciones } \\
\text { - Cuidar horario apropiado }\end{array}$ & \begin{tabular}{|lr|}
\multicolumn{2}{|l|}{ - Autoinstrucciones } \\
- Activación r condicionada \\
(activación r conseguida \\
durante los entrenamientos)
\end{tabular} & $\begin{array}{l}\text { - Dormir, reposar } \\
\text { - Ejercicio físico para eliminar tensión } \\
\text { - Relajación } \\
\text { - Autoinstrucciones }\end{array}$ & $\begin{array}{ll}\text { - } & \text { Relajación condicionada } \\
\text { - } & \text { Respiración profunda } \\
\text { - } & \text { Autoinstrucciones }\end{array}$ \\
\hline $\begin{array}{l}\text { ACTIVACION } \\
\text { COGNITIVA } \\
\text { POSITIVA } \\
\text { (MOTIVACIÒN) }\end{array}$ & $\begin{array}{ll}\text { - } & \begin{array}{l}\text { Establecimiento de objetivos concretos } \\
\text { especiales }\end{array} \\
\text { - } & \text { Elaborar un plan para la competición con } \\
\text { objetivos prioritarios } \\
\text { - } & \text { Concentrarse en esos objetivos } \\
\text { - } & \text { Repasar imágenes positivas respecto a su } \\
\text { propio rendimiento } & \text { Pensar en el éxito en la competición y e las } \\
\text { - } & \text { consecuencias del mismo } \\
\text { Recordar situaciones pasadas similares que } \\
\text { hayan dejado un recuerdo positivo. }\end{array}$ & $\begin{array}{ll}\text { - } & \text { Palabras de ánimo } \\
\text { - } & \text { Pensamientos positivos } \\
\text { - } & \text { Concentración de y } \\
& \text { visualización de los } \\
& \text { objetivos positivos. } \\
\text { - } & \text { Autoinstrucciones. }\end{array}$ & \begin{tabular}{|l} 
- \\
- Detención de pensamientos activadores \\
- \\
Situarse en una perspectiva realista \\
respecto a la competición (importancia \\
de esta en un contexto más amplio, \\
objetivos reales de la misma, \\
posibilidades reales de éxito, etc.) \\
Elaborar un plan realista para afrontar la \\
competición estableciendo objetivos \\
prioritarios.
\end{tabular} & $\begin{array}{ll}\text { - } & \text { Detención de pensamientos } \\
\text { - } & \text { Autoinstrucciones } \\
\text { - } & \text { Concentración en los objetivos } \\
\text { prioritarios de esa competición. } \\
\text { - } \quad \text { Apartar la concentración del } \\
\text { Resultado de la competición, } \\
\text { centrarse en lo más próximo (Ej. } \\
\text { Realizar una buena salida.) }\end{array}$ \\
\hline $\begin{array}{l}\text { ACTIVACION } \\
\text { COGNITIVA } \\
\text { NEGATIVA } \\
\text { (ESTRÈS) }\end{array}$ & $\begin{array}{l}\text { - Que realice pensamientos en relación con la } \\
\text { dificultad de la competición, los puntos } \\
\text { fuertes y débiles de los rivales, la } \\
\text { posibilidad de fracasar y las consecuencias } \\
\text { de un fracaso, etc. } \\
\text { - Pensar en las posibles consecuencias } \\
\text { negativas de competir con un nivel de } \\
\text { activación bajo. } \\
\text { Recordar situaciones pasadas similares en } \\
\text { las que se fracasó o que han dejado un } \\
\text { impacto negativo. } \\
\text { Pensar en la posible evaluación negativa de } \\
\text { personas relevantes para él (amigos/as, } \\
\text { entrenador, padres, ...) } \\
\text { Anticipar problemas reales que pueden } \\
\text { surgir o presentarse durante la competición. }\end{array}$ & 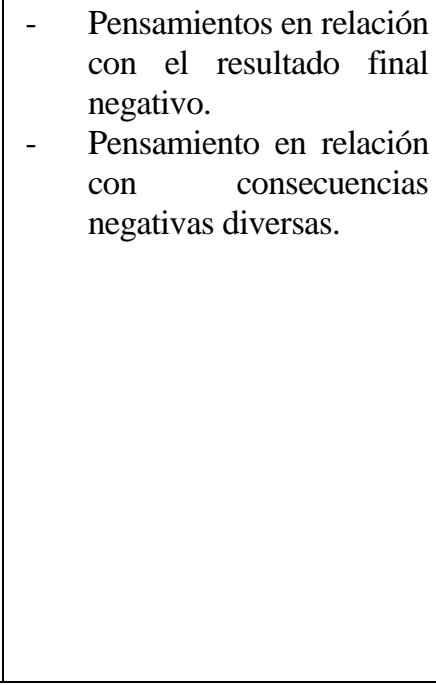 & 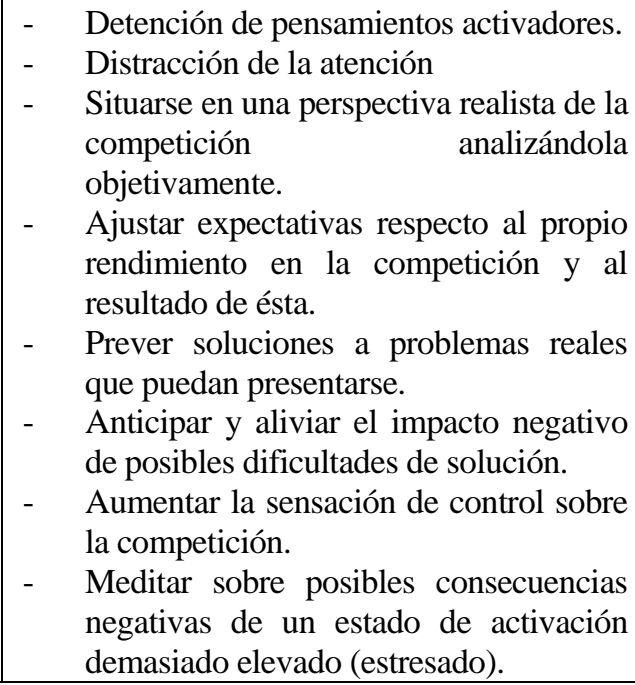 & $\begin{array}{ll}\text { - } & \text { Detención de pensamientos } \\
\text { negativos } \\
\text { - } & \text { Autoinstrucciones } \\
\text { - } & \text { Apartar la concentración de } \\
\text { cuestiones que no dependen del } \\
\text { propio deportista. } \\
\text { - Apartar la concentración del } \\
\text { resultado final de la competición } \\
\text { - Aceptar dificultades e intentar } \\
\text { aliviar solucionarlas o reducir su } \\
\text { impacto. }\end{array}$ \\
\hline
\end{tabular}

